\title{
Global dynamics of triangular maps
}

\author{
E. Cabral Balreira ${ }^{a}$, Saber Elaydi ${ }^{a, b, *}$, Rafael Luís ${ }^{b}$ \\ a Department of Mathematics, Trinity University, San Antonio, TX, USA \\ ${ }^{\mathrm{b}}$ Center for Mathematical Analysis, Geometry, and Dynamical Systems, Instituto Superior Tecnico, Technical University of Lisbon, \\ Lisbon, Portugal
}

\section{A R T I C L E I N F O}

\section{Article history:}

Received 30 November 2013

Accepted 26 March 2014

Communicated by S. Carl

\section{MSC:}

$37 C 75$

39A30

Keywords:

Triangular maps

Sharkovsky's Theorem

Omega limit set

Orbit

Fiber

Global stability

\begin{abstract}
A B S T R A C T
We consider continuous triangular maps on $I^{N}$, where $I$ is a compact interval in the Euclidean space $\mathbb{R}$. We show, under some conditions, that the orbit of every point in a triangular map converges to a fixed point if and only if there is no periodic orbit of prime period two. As a consequence we obtain a result on global stability, namely, if there are no periodic orbits of prime period 2 and the triangular map has a unique fixed point, then the fixed point is globally asymptotically stable. We also discuss examples and applications of our results to competition models.
\end{abstract}

(c) 2014 Elsevier Ltd. All rights reserved.

\section{Introduction}

Global dynamics and global stability of dynamical systems in higher dimension are some of the most challenging problems in Mathematics. An important problem in this area is the LaSalle [1] conjecture which states that if all the eigenvalues of the Jacobian matrix of a map have modulus less than one at every point in the domain, then the fixed point of the system is globally asymptotically stable. It turns out that LaSalle's conjecture is false even for planar maps, as shown independently by Chamberland [2] and Martelli [3]. In its essence, LaSalle's conjecture strives to find conditions under which a fixed point of a map is globally asymptotically stable.

Realizing that unimodal one dimensional maps and most of planar population models do not satisfy the stringent condition on the eigenvalues of the Jacobian matrix of a map, the authors developed a different approach by Balreira, Elaydi and Luís [4] to global asymptotic stability. The authors showed that if the unique fixed point of the planar Ricker competition model is locally asymptotically stable then, under minor conditions, it is globally asymptotically stable. Note that to ensure local asymptotic stability of a fixed point, it is sufficient to show that the modulus of the eigenvalues of the Jacobian is less than one in a small neighborhood of the fixed point.

It is trivially true that if a map has a fixed point that is globally asymptotically stable, then there are no periodic orbits of prime period greater than or equal to two. In one dimension, [5] have shown that the converse is also true provided that the orbits are bounded and there is only one fixed point. One may consider if this also holds in higher dimension. In this article, we show that for a class of maps, called triangular, this result may be extended provided that all the orbits are bounded.

\footnotetext{
* Corresponding author at: Department of Mathematics, Trinity University, San Antonio, TX, USA. Tel.: +1 210736 8246.

E-mail addresses: ebalreir@trinity.edu (E. Cabral Balreira), selaydi@trinity.edu (S. Elaydi), rafael.luis.madeira@gmail.com (R. Luís).
} 
The significance of our result is due to the fact that our conditions for global asymptotic stability are easily verifiable. Moreover, our main result, Theorem 3.1, is much more general since it establishes the global dynamics of triangular maps that possess more than just one fixed point. It should be noted that results on the global dynamics of planar monotone maps were established by Smith [6]. In addition, similar results were also obtained by Kulenović and Merino [7-9] who studied the global dynamics of planar competitive and cooperative planar systems. Moreover, the work by Amleh, Camouzis and Ladas [10] established global stability results of second order rational difference equations.

An important tool needed in the sequel is Sharkovsky's Theorem. Established by Sharkovsky [11], this results has played a role of paramount importance in the dynamics of one-dimensional maps. In higher dimensions, [12] has shown that Sharkovsky's Theorem also holds for triangular maps. However it was not until recent work on stability of Balreira, Elaydi and Luís [4] and Guzowska, Luís and Elaydi [13] that a better understanding of the stable and unstable manifold yielded the appropriate setting to generalize the work by Elaydi and Sacker [5] and establish our results on global asymptotically stability and global dynamics.

We organize the paper as follows. In Section 2 we introduce the notation and discuss the known and relevant results to this work. In Section 3 we proof the main result of this note and establish a result on global asymptotically stability. Finally, in Section 4 we provide examples of triangular maps, including the triangular Leslie-Gower competition model, to illustrate our results. In addition, we given an in depth analysis of the logistic triangular map on $I^{N}$, where $I=[0,1]$, is provided.

\section{Preliminaries}

We begin with a discussion of the materials and concepts that will be used in this paper. Let us now introduce the main object of study in this work.

Definition 2.1. A map $F: \mathbb{R}^{N} \rightarrow \mathbb{R}^{N}$ is said to be triangular if it can be written as

$$
F\left(x_{1}, x_{2}, \ldots, x_{N}\right)=\left(f_{1}\left(x_{1}\right), f_{2}\left(x_{1}, x_{2}\right), \ldots, f_{N}\left(x_{1}, x_{2}, \ldots, x_{N}\right)\right) .
$$

For a point $\mathbf{x} \in \mathbb{R}^{N}$, the orbit of $\mathbf{x}$ under $F$ is given by

$$
O(\mathbf{x})=\left\{F^{n}(\mathbf{x}) \mid n \in \mathbb{Z}^{+}\right\},
$$

where $\mathbb{Z}^{+}$is the set of nonnegative integers. The omega limit set $\omega(\mathbf{x})$ of $\mathbf{x} \in \mathbb{R}^{N}$ is defined as

$$
\omega(\mathbf{x})=\left\{z \in \mathbb{R}^{N} \mid F^{n_{i}}(\mathbf{x}) \rightarrow z, \text { as } n_{i} \rightarrow \infty\right\},
$$

for some subsequence $n_{i}$ of $\mathbb{Z}^{+}$. The set $\omega(\mathbf{x})$ is closed and invariant and it is non-empty if the orbit closure of $\mathbf{x}$ is compact.

In the case of one dimensional maps, [5] have shown that if a map does not have a periodic orbit, then the orbit of every point converges to a fixed point. Namely, they have shown the following result.

Theorem 2.2 ([5]). Let $I=[a, b] \subseteq \mathbb{R}$ and $f: I \rightarrow I$ be a continuous map. If $f$ has no points of prime period two, then every orbit under the map $f$ converges to a fixed point.

It should be noted that Theorem 2.2 is also true if the interval $I$ is replaced by $\mathbb{R}^{+}=[0, \infty)$ but under the assumption that all orbits are bounded. The proof of Theorem 2.2 relies on the celebrated Sharkovsky's Theorem for one-dimensional dynamical systems. Sharkovsky [11] introduces an ordering $\triangleright$ of the positive integers which we indicate below

$$
\begin{aligned}
& 3 \triangleright 5 \triangleright 7 \triangleright 9 \triangleright \cdots \triangleright(2 n+1) \times 2^{0} \triangleright \cdots \\
& 3 \times 2 \triangleright 5 \times 2 \triangleright 7 \times 2 \triangleright 9 \times 2 \triangleright \cdots \triangleright(2 n+1) \times 2^{1} \triangleright \cdots \\
& \vdots \\
& 3 \times 2^{n} \triangleright 5 \times 2^{n} \triangleright 7 \times 2^{n} \triangleright 9 \times 2^{n} \triangleright \cdots \triangleright(2 n+1) \times 2^{n} \triangleright \cdots \\
& \vdots \\
& \cdots \triangleright 2^{n} \triangleright 2^{n-1} \triangleright \cdots \triangleright 2^{3} \triangleright 2^{2} \triangleright 2^{1} \triangleright 2^{0} .
\end{aligned}
$$

Then Sharkovsky's Theorem is as follows.

Theorem 2.3 ([11]). Let $F: I \rightarrow$ I be a continuous map which has a periodic orbit of prime period $k$. Then for any positive integer $l$ that is preceded by $k$ in the Sharkovsky order $k \triangleright l$, there is a periodic orbit of prime period $l$.

We note that Theorem 2.3 is a one-dimensional result and, in general, it is not known if it holds in higher dimensions. However, if one considers triangular maps, [12] showed that Sharkovsky's Theorem holds true.

Theorem 2.4 ([12]). Let $F: I^{N} \rightarrow I^{N}$ be a continuous triangular map. Then the conclusion of Sharkovsky's Theorem holds true.

In our work, we will focus on the generalization of Theorem 2.2 for triangular maps with domain and codomain to be $N$-dimensional cubes, that is, $I^{N}$ where $I=[a, b] \subset \mathbb{R}$. We note that in applications to population models, where the domain is the nonnegative orthant $\mathbb{R}_{+}^{N}$, we shall add the assumption that all orbits are bounded and our results will remain valid. 
Another interest of the results in this paper are on the investigation of global stability of fixed points. Indeed, let us consider the map $F: I^{N} \rightarrow I^{N}$ of class $C^{1}$ and let $\mathbf{x}^{*}$ be a fixed point of the map $F$. We say that $\mathbf{x}^{*}$ is stable if for any $\varepsilon>0$, there is $\delta>0$ such that for all $\mathbf{x}_{0} \in I^{N}$ with $\left\|\mathbf{x}_{0}-\mathbf{x}^{*}\right\|<\delta$ we have $\left\|F\left(\mathbf{x}_{0}\right)-F\left(\mathbf{x}^{*}\right)\right\|<\varepsilon$. In addition, $\mathbf{x}^{*}$ is attracting if there is $\eta>0$ such that $\left\|\mathbf{x}_{0}-\mathbf{x}^{*}\right\|<\eta$ implies $\lim _{n \rightarrow \infty} F^{n}\left(\mathbf{x}_{0}\right)=\mathbf{x}^{*}$. Finally, $\mathbf{x}^{*}$ is asymptotically stable if it is both stable and attracting. Observe that such definitions are local, that is, only need to be satisfied on a neighborhood of $\mathbf{x}^{*}$. Thus we say that $\mathbf{x}^{*}$ is globally asymptotically stable if the above definition holds for all $\eta$.

Analytically, these questions, at least locally, are often considered by looking at the eigenvalues of the Jacobian matrix of $F$ at $\mathbf{x}^{*}$, denoted by $J F\left(\mathbf{x}^{*}\right)$. Indeed, let us define $\sigma\left(\mathbf{x}^{*}\right)=\left\{\lambda \in \mathbb{C} \mid \lambda\right.$ is an eigenvalue of $\left.J F\left(\mathbf{x}^{*}\right)\right\}$. When clear from context, we will simply denote $\sigma\left(\mathbf{x}^{*}\right)$ as $\sigma$. We have that $\sigma$ decomposes as the following subsets.

$$
\begin{aligned}
& \sigma^{s}=\{\lambda \in \mathbb{C}|| \lambda \mid<1\}, \\
& \sigma^{c}=\{\lambda \in \mathbb{C}|| \lambda \mid=1\}, \\
& \sigma^{u}=\{\lambda \in \mathbb{C}|| \lambda \mid>1\} .
\end{aligned}
$$

For each of the subsets above, the tangent space of $\mathbf{x}^{*}$ is decomposed into the invariant subspaces $E^{s}, E^{c}, E^{u}$ corresponding to $\sigma^{s}, \sigma^{c}, \sigma^{u}$, that is, $\mathbb{R}^{N}=E^{s} \oplus E^{c} \oplus E^{u}$. It should be noted that some of these subspaces may be trivial subspaces.

Given an open neighborhood $U$ of $\mathbf{x}^{*}$, the local stable manifold for $\mathbf{x}^{*}$ in this neighborhood is defined to be the set

$$
W_{l o c}^{s}\left(\mathbf{x}^{*}, U\right)=\left\{z \in U \mid \lim _{n \rightarrow \infty} F^{n}(z)=\mathbf{x}^{*}\right\}
$$

and the local unstable manifold for $\mathbf{x}^{*}$ in the neighborhood $U$ is the set

$$
W_{l o c}^{u}\left(\mathbf{x}^{*}, U\right)=\left\{z \in U \mid \text { there exists a complete negative orbit }\left\{\mathbf{x}_{-n}^{*}\right\} \subset U \text { such that } \lim _{n \rightarrow \infty} \mathbf{x}_{-n}^{*}=\mathbf{x}^{*}\right\} .
$$

The stable manifold theory $[14,15]$ guarantees the existence of the local stable and unstable manifolds in a suitable open neighborhood $U$ of the fixed point $\mathbf{x}^{*}$. In this case, we denote the stable, center, and unstable manifolds of $\mathbf{x}^{*}$ by $W_{l o c}^{s}\left(\mathbf{x}^{*}\right)$, $W_{l o c}^{c}\left(\mathbf{x}^{*}\right), W_{l o c}^{u}\left(\mathbf{x}^{*}\right)$, respectively. Moreover, all these manifolds are invariant.

Once we have the local unstable manifold, then the global unstable manifold is given by

$$
W^{u}\left(\mathbf{x}^{*}\right)=\bigcup_{n \geq 0} F^{n}\left(W_{l o c}^{u}\left(\mathbf{x}^{*}\right)\right) .
$$

It should be noted that since $F$ is of class $C^{1}$, both $W_{l o c}^{u}$ and $W_{l o c}^{s}$ are $C^{1}$ manifolds.

\section{Main results}

We consider the dynamics of triangular maps, as defined in 2.1 with domain and codomain $I^{N} \subseteq \mathbb{R}^{n}$, where $I=[a, b]$. Our main result generalizes Theorem 2.2 and has applications to determine global stability. The main theorem is as follows.

Theorem 3.1. Let $F: I^{N} \rightarrow I^{N}$ be a continuous $C^{1}$ triangular map such that each fixed points $\mathbf{x}^{*}$ is a locally stable fixed points of $\left.F\right|_{W_{l o c}^{c}} ^{c}\left(\mathbf{x}^{*}\right)$. Then every orbit in $I^{N}$ converge to a fixed point if and only if there are no periodic orbits of prime period two.

We remark that if a fixed point $\mathbf{x}^{*}$ is hyperbolic, i.e., none of the fixed points are on the unit circle, then the condition on the center manifold, that is, the fixed point $\mathbf{x}^{*}$ is a locally stable fixed points of $\left.F\right|_{W_{l o c}^{c}\left(\mathbf{x}^{*}\right)}$, is not needed. An important consequence of Theorem 3.1, is the following result establishing global stability.

Corollary 3.2. Suppose that the triangular map $F$ has a unique fixed point $\mathbf{x}^{*}$ and $\mathbf{x}^{*}$ is stable for $\left.F\right|_{W_{l o c}^{c}\left(\mathbf{x}^{*}\right)}$. Then $\mathbf{x}^{*}$ is globally asymptotically stable if and only if $F$ has no periodic orbits of prime period two.

The proof of our main result, Theorem 3.1, is obtained by taking advantage of the structure of our triangular maps. First, let us establish some lemmas that will be used in our proof.

Lemma 3.3. If the triangular map $F=\left(f_{1}, f_{2}, \ldots, f_{N}\right): I^{N} \rightarrow I^{N}$ has no periodic orbits of period 2 , then the map $f_{1}$ has no periodic orbits of prime period greater than one.

Proof. Assume that $F$ has no periodic orbits of period two. Since Sharkovsky's order is valid for triangular maps, see Theorem 2.4, we conclude that $F$ has no periodic orbits of any prime period greater than one. Let us now suppose towards a contradiction that $f_{1}$ has a periodic orbit of prime period $r>1$, we will show that this will induce a periodic orbit of $F$ which is the desired contradiction.

Using the Sharkovsky order, we conclude by Theorem 2.3 that $f_{1}$ must have a periodic orbit of prime period two, say that this orbit is $\left\{a_{11}, a_{12}\right\}$. Note that $a_{11}$ is a fixed point under the composition map $f_{1}^{2}=f_{1} \circ f_{1}$, and for simplicity we will denote such fixed point by $a_{1}^{*}$.

Next, we consider the component map $f_{2}: I^{2} \rightarrow I$ and define the following maps $f_{21}, f_{22}: I \rightarrow I$ by

$$
f_{21}(z)=f_{2}\left(a_{11}, z\right) \text { and } f_{22}(z)=f_{2}\left(a_{12}, z\right) \text {. }
$$


Now consider the composition map $\widehat{f}_{2}=f_{22} \circ f_{21}$. From Theorem 2.2 we have that either $\widehat{f}_{2}$ has a periodic orbit of prime period two or every orbit converges to a fixed point of $\widehat{f}_{2}$.

In the later case, if the orbits of $f_{2}$ converges to a fixed point, we pick one of these fixed points and denote it by $a_{2}^{*}$. Observe that trivially, $a_{2}^{*}$ is a periodic orbit of $\widehat{f}_{2}$. In the case that $\widehat{f}_{2}$ has a periodic orbit of prime period two, say $\left\{a_{21}, a_{22}\right\}$, we have that $\widehat{f}_{2}^{2}$ has a fixed point, without lost of generality, we denoted it as $a_{2}^{*}$.

For notational purposes, let us denote the orbit of $\left\{a_{21}, a_{22}\right\}$ by the maps $f_{21}$ and $f_{22}$ as

$$
f_{21}\left(a_{21}\right)=\widehat{a}_{21}, \quad f_{22}\left(\widehat{a}_{21}\right)=a_{22}, \quad f_{22}\left(a_{22}\right)=\widehat{a}_{22}, \quad f_{22}\left(\widehat{a}_{22}\right)=a_{21} .
$$

Observe that if one considers the map $F_{12}: I^{2} \rightarrow I^{2}$ to be given by $F_{12}\left(x_{1}, x_{2}\right)=\left(f_{1}\left(x_{1}\right), f_{2}\left(x_{1}, x_{2}\right)\right)$, then $F_{12}^{4}\left(a_{11}, a_{21}\right)=$ $\left(a_{11}, a_{21}\right)$, i.e., the map $F_{12}$ obtained by the first two components of $F$ is periodic.

Next, as we proceed to analyze the component $\operatorname{map} f_{3}: I^{3} \rightarrow I$ and we define the four maps as follows:

$$
\begin{aligned}
& f_{31}(z)=f_{3}\left(a_{11}, a_{21}, z\right), \quad f_{32}(z)=f_{3}\left(a_{12}, \widehat{a}_{21}, z\right), \\
& f_{33}(z)=f_{3}\left(a_{11}, a_{22}, z\right), \quad \text { and } f_{34}(z)=f_{3}\left(a_{12}, \widehat{a}_{22}, z\right) .
\end{aligned}
$$

Consider their composition $\widehat{f}_{3}=f_{34} \circ f_{33} \circ f_{32} \circ f_{31}$. Similarly to the reasoning above $\widehat{f}_{3}$ either has a periodic orbit of prime period two or every orbit converges to a fixed point. Again, by analogous process, either case produces a periodic orbit of $\widehat{f}_{3}$, that is, the map $\widehat{f}_{3}^{m}$ has a fixed point for some $m>1$. Hence the map obtained by the first three components of $F$ is periodic. Repeating this process $N$ times, we obtain the point $\left(a_{1}^{*}, a_{2}^{*}, \ldots, a_{N}^{*}\right)$ which is a fixed point of the map $F^{k}$, for some positive integer $k>1$, that is, we obtain a periodic point of $F$ which is the contradiction we seek. Therefore the map $f_{1}$ has no periodic orbits of prime period 2 and by Sharkovsky's Theorem, we conclude that $f_{1}$ has no periodic orbits of any prime period greater than one.

As we proceed with our goal to prove Theorem 3.1, we observe that Lemma 3.3 allows us to conclude that the first component map $f_{1}$ does not have a periodic point if the map $F$ does not have a periodic orbit. This implies by Theorem 2.2 that one could simply work on the fiber of each fixed point of the map $f_{1}$.

In the sequel, we will need the following result of Brunovský and Poláčik [16].

Theorem 3.4 ([16]). Let $\mathbf{x}^{*}$ be a fixed point of the map F such that $\mathbf{x}^{*} \in \omega(\mathbf{x})$ for some point $\mathbf{x} \in I^{N}$. Assume further that $\mathbf{x}^{*}$ is stable for $\left.F\right|_{W_{l o c}^{c}\left(\mathbf{x}^{*}\right)}$. Then either $\lim _{n \rightarrow \infty} F^{n}(\mathbf{x})=\mathbf{x}^{*}$ or else $\omega(\mathbf{x})$ contains a point of $W_{\text {loc }}^{u}\left(\mathbf{x}^{*}\right) \backslash\left\{\mathbf{x}^{*}\right\}$.

Observe that if the omega limiting set consists of a set of fixed points, then a similar result to Theorem B by Brunovský and Poláčik [16] was proven by Aulbach [17].

In addition, if the local center manifold $W_{l o c}^{c}\left(\mathbf{x}^{*}\right)$ of a fixed point is one-dimensional, then one may provide conditions for $\mathbf{x}^{*}$ to be stable for $\left.F\right|_{W_{l o c}^{c}}\left(\mathbf{x}^{*}\right)$. For instance, let $f=\left.F\right|_{W_{l o c}^{c}}\left(\mathbf{x}^{*}\right)$. If $f^{\prime}\left(\mathbf{x}^{*}\right)=1$, then $\mathbf{x}^{*}$ is stable for $\left.F\right|_{W_{l o c}^{c}}\left(\mathbf{x}^{*}\right)$ if $f^{\prime \prime}\left(\mathbf{x}^{*}\right)=0$ and $f^{\prime \prime \prime}\left(\mathbf{x}^{*}\right)<0$. On the other hand, if $f^{\prime}\left(\mathbf{x}^{*}\right)=-1$, then $\mathbf{x}^{*}$ is stable for $\left.F\right|_{W_{\text {loc }}^{c}}\left(\mathbf{x}^{*}\right)$ if the Schwarzian derivative $(S f)\left(\mathbf{x}^{*}\right)<0$, where

$$
(S f)\left(\mathbf{x}^{*}\right)=\frac{f^{\prime \prime \prime}\left(\mathbf{x}^{*}\right)}{f^{\prime}\left(\mathbf{x}^{*}\right)}-\frac{3}{2}\left(\frac{f^{\prime \prime}\left(\mathbf{x}^{*}\right)}{f^{\prime}\left(\mathbf{x}^{*}\right)}\right)^{2} .
$$

Proof of Theorem 3.1. Let $\mathbf{x}=\left(x_{1}, x_{2}, \ldots, x_{N}\right) \in I^{N}$. From the assumption that $F$ has no periodic orbits of prime period 2, we conclude by Lemma 3.3 that the coordinate map $f_{1}$ has no periodic orbits of prime period 2 . Therefore, by Theorem 2.2 we must have

$$
\lim _{n \rightarrow \infty} f_{1}^{n}\left(x_{1}\right)=x_{1}^{*},
$$

for some fixed point $x_{1}^{*}$ of the $f_{1}$. Let us now consider the map $F_{2}: I^{2} \rightarrow I^{2}$ given by

$$
F_{2}(u, v)=\left(f_{1}(u), f_{2}(u, v)\right) .
$$

From the discussion above, the omega limit set $\omega\left(x_{1}, x_{2}\right)$ under $F_{2}$ will be contained in the fiber $\left\{\left(x_{1}^{*}, t\right) \mid t \in I\right\}$. By compactness, there exists $\left(x_{1}^{*}, y_{2}\right) \in \omega\left(x_{1}, x_{2}\right)$. Next, define the map $\widehat{f}_{2}: I \rightarrow I$ given by $\widehat{f}_{2}(t)=f_{2}\left(x_{1}^{*}, t\right)$, that is, the map $f_{2}$ restricted to the fiber of $I^{2}$ with first component $x_{1}^{*}$. An analogous argument of Lemma 3.3 with the restriction of $F$ to the hypersurface $\mathscr{H}_{x_{1}^{*}}=\left\{\left(x_{1}, x_{2}, \ldots, x_{N}\right) \in I^{N} \mid x_{1}=x_{1}^{*}\right\}$ establishes that if $F$ has no periodic orbits of prime period 2 , then neither does the map $\widehat{f}_{2}$. Using Theorem 2.2 applied to the point $y_{2}$, we obtain that

$$
\lim _{n \rightarrow \infty} \widehat{f}_{2}^{n}\left(y_{2}\right)=x_{2}^{*} \text {, }
$$

where $x_{2}^{*}$ is a fixed point of $\widehat{f}_{2}$. Hence $\left(x_{1}^{*}, x_{2}^{*}\right)$ is a fixed point of the map $F_{2}$ and we have that $\left(x_{1}^{*}, x_{2}^{*}\right) \in \omega\left(x_{1}^{*}, y_{2}\right)$. Since the omega limiting set is closed and invariant, see [14,15], we must have that $\left(x_{1}^{*}, x_{2}^{*}\right) \in \omega\left(x_{1}, x_{2}\right)$ and the map $F_{2}$ satisfies the hypotheses of Theorem 3.4. Therefore, we have that either $\lim _{n \rightarrow \infty} F_{2}^{n}\left(x_{1}, x_{2}\right)=\left(x_{1}^{*}, x_{2}^{*}\right)$ or there exists $z=\left(z_{1}, z_{2}\right) \in \omega\left(x_{1}, x_{2}\right) \bigcap W_{l o c}^{u}\left(x_{1}^{*}, x_{2}^{*}\right)$. 
Assume that the second alternative holds, that is, there is a point $z=\left(z_{1}, z_{2}\right) \in \omega\left(x_{1}, x_{2}\right) \bigcap W_{l o c}^{u}\left(x_{1}^{*}, x_{2}^{*}\right)$. From (2) we have that $\omega(X) \subseteq \mathscr{H}_{x_{1}^{*}}$ and in particular $z_{1}=x_{1}^{*}$.

Now consider the dynamics of the point $z=\left(x_{1}^{*}, z_{2}\right)$ under the map $F_{2}$. Since $x_{1}^{*}$ is a fixed point of $f_{1}$, the global unstable manifold of $\left(x_{1}^{*}, x_{2}^{*}\right)$ belongs to the fiber $\left\{\left(x_{1}^{*}, t\right) \in I^{2}\right\}$ and it can be viewed as a bounded interval on this fiber because the orbits of $F$ are, by hypotheses, bounded. In addition, the endpoints of $W^{u}\left(x_{1}^{*}, x_{2}^{*}\right)$ must be fixed points of $F_{2}$ and without loss of generality, we have

$$
\lim _{n \rightarrow \infty} F_{2}^{n}\left(x_{1}^{*}, z_{2}\right)=\left(x_{1}^{*}, z_{2}^{*}\right)
$$

where $\left(x_{1}^{*}, z_{2}^{*}\right)$ is an endpoint of $W^{u}\left(x_{1}^{*}, x_{2}^{*}\right)$ and a fixed point of $F_{2}$. In fact, we can also say a bit more about $\left(x_{1}^{*}, z_{2}^{*}\right)$. First, there is a neighborhood $U$ of $\left(x_{1}^{*}, z_{2}^{*}\right)$ such that $U \cap W^{u}\left(x_{1}^{*}, z_{2}^{*}\right)=W_{l o c}^{s}\left(x_{1}^{*}, z_{2}^{*}\right)$, that is, near $\left(x_{1}^{*}, z_{2}^{*}\right)$, the global unstable manifold of $\left(x_{1}^{*}, x_{2}^{*}\right)$ contains the locally stable manifold of $\left(x_{1}^{*}, z_{2}^{*}\right)$. Secondly, $\left(x_{1}^{*}, z_{2}^{*}\right) \in \omega\left(x_{1}, x_{2}\right)$ as $\omega\left(x_{1}, x_{2}\right)$ is closed and invariant and $\left(x_{1}^{*}, z_{2}\right) \in \omega\left(x_{1}, x_{2}\right)$ by our assumption above.

Therefore, we can again apply Theorem 3.4 with respect to the fixed point $\left(x_{1}^{*}, z_{2}^{*}\right)$ and conclude that either $\lim _{n \rightarrow \infty} F_{2}^{n}\left(x_{1}, x_{2}\right)=\left(x_{1}^{*}, z_{2}^{*}\right)$ or there exists $z^{\prime}=\left(z_{1}^{\prime}, z_{2}^{\prime}\right) \in \omega\left(x_{1}, x_{2}\right) \bigcap W_{\text {loc }}^{u}\left(x_{1}^{*}, z_{2}^{*}\right)$.

However, the second alternative leads to a contradiction since $\left(x_{1}^{*}, z_{2}^{*}\right)$ cannot have a local unstable manifold. Indeed, by the argument above it has a locally stable manifold and in order to $W_{l o c}^{u}\left(x_{1}^{*}, z_{2}^{*}\right)$ exist we must have that $\left(x_{1}^{*}, z_{2}^{*}\right)$ is semi-stable which cannot occur by the hypotheses of our theorem. Thus, $\left(x_{1}, x_{2}\right)$ must converge to a fixed point of $F_{2}$, and for simplicity of notation, we assume that

$$
\lim _{n \rightarrow \infty} F_{2}^{n}\left(x_{1}, x_{2}\right)=\left(x_{1}^{*}, x_{2}^{*}\right) \text {. }
$$

Next, define $F_{3}: I^{3} \rightarrow I^{3}$ by letting

$$
F_{3}\left(z_{1}, z_{2}, z_{3}\right)=\left(f_{1}\left(z_{1}\right), f_{2}\left(z_{1}, z_{2}\right), f_{3}\left(z_{1}, z_{2}, z_{3}\right)\right),
$$

and consider the point $\left(x_{1}, x_{2}, x_{3}\right)$. Then using an argument similar to the one used above, we consider the map $\widehat{f}_{3}: I \rightarrow I$ to be $\widehat{f}_{3}(t)=f_{3}\left(x_{1}^{*}, x_{2}^{*}, t\right)$, that is, the map $f_{3}$ restricted to the fiber of $I^{3}$ with the first two coordinates $\left(x_{1}^{*}, x_{2}^{*}\right)$. We can then show that

$$
\lim _{n \rightarrow \infty} F_{3}^{n}\left(x_{1}, x_{2}, x_{3}\right)=\left(x_{1}^{*}, x_{2}^{*}, x_{3}^{*}\right),
$$

where $\left(x_{1}^{*}, x_{2}^{*}, x_{3}^{*}\right)$ is a fixed point of $F_{3}$. Iterating this process, one may show that the orbit of the point $\left(x_{1}, x_{2}, \ldots, x_{N}\right)$ converges to a fixed point of the map $F$.

\section{Illustrative examples}

In this section we present some examples in order to better show how our results can be applied.

Example 4.1. Consider the triangular map defined on $\mathbb{R}_{+}^{2}$ given by

$$
F(x, y)=(a x, y(1+x)), \quad \text { with }|a|<1 .
$$

The map $F$ has a 2-periodic solution whenever $F(F(x, y))=(x, y)$, or equivalently,

$$
a^{2} x=x \text { and } y(1+x)(1+a x)=y .
$$

The only solution of the system $(3)$ is $(0, y)$ since $|a|<1$. In this case, we see that $(0, y)$ is also a fixed point and thus the map $F$ has no periodic orbits of prime period two. We observe that the orbits of $F$ are bounded. Indeed, for any $\left(x_{0}, y_{0}\right) \in \mathbb{R}_{+}^{2}$, we have that

$$
F^{n}\left(x_{0}, y_{0}\right)=\left(a^{n} x_{0}, y_{0} \prod_{j=0}^{n-1}\left(1+a^{j} x_{0}\right)\right) .
$$

The first component is bounded as $\left|a^{n} x_{0}\right|<\left|x_{0}\right|$ while the second component is bounded because the infinite product $\prod_{j=0}^{\infty}\left(1+a^{j} x_{0}\right)$ converges to a limit, say $\beta>0$. Indeed, since $\sum_{n=0}^{\infty} a^{n}$ converges one can show, see for instance [18, pp. 345] that

$$
\lim _{n \rightarrow \infty} \prod_{j=0}^{n}\left(1+a^{j} x_{0}\right)=\beta y_{0}
$$

Hence the orbits of $F$ are bounded. Next, we compute $\sigma(0, y)=\{a, 1\}$. Thus in order to satisfy the hypotheses of Theorem 3.1, we need to show that on the center manifold the fixed point $(0, y)$ is stable. In fact, using the techniques employed in [19], we can show that $W_{l o c}^{c}(0, y)$ is the $y$-axis. Since every point in the $y$-axis is fixed, then $(0, y)$ is stable for $\left.F\right|_{W_{l o c}^{c}}(0, y)$. Thus all the hypotheses of Theorem 3.1 are satisfied and therefore every orbit converges to a fixed point. 
Remark 4.2. A simple modification of Example 4.1 shows that $F$ may have a periodic orbit of prime period 2. Indeed, consider the triangular map defined on $\mathbb{R}_{+}^{2}$ given by

$$
F(x, y)=(a x, y(-1+x)), \quad \text { with }|a|<1 .
$$

Analogous computation shows that the map $F$ has a 2-periodic solution for $(0, y)$ and if $y \neq 0$, then $(0, y)$ is a periodic point of prime period two. In this case for $\left(x_{0}, y_{0}\right)$, we have

$$
F^{n}\left(x_{0}, y_{0}\right)=\left(a^{n} x_{0},(-1)^{n}\left[\prod_{j=0}^{n-1}\left(1+a^{n} x_{0}\right)\right] y_{0}\right) \text {. }
$$

As aforementioned, when $|a|<1, \prod_{j=0}^{\infty}\left(1+a^{n} x_{0}\right)=\gamma$, for some $\gamma \in \mathbb{R}$ then

$$
\lim _{n \rightarrow \infty} y_{n}= \begin{cases}\gamma y_{0}, & \text { if } n \text { is even } \\ -\gamma y_{0}, & \text { if } n \text { is odd. }\end{cases}
$$

Hence, every orbit of a point $\left(x_{0}, y_{0}\right) \in \mathbb{R}^{2}$ converges to a periodic orbit on the fiber $x^{*}=0$.

Remark 4.3. Another modification of Example 4.1, shows that it is possible for unbounded orbits to exist. For instance, let

$$
F(x, y)=\left(\frac{\mu K x}{K+(\mu-1) x}, y(1+x)\right),
$$

with $\mu>1, K>0$, that is, the first component be given by the Beverton-Holt model. Now, for any $\left(x_{0}, y_{0}\right) \in \mathbb{R}_{+}^{2}$, let $F^{n}\left(x_{0}, y_{0}\right)=\left(x_{n}, y_{n}\right)$, then $\lim _{n \rightarrow \infty} x_{n}=K>0$. However, as we consider the second component, we see that $\prod_{n=0}^{\infty}\left(1+x_{n}\right)=\infty$ and all solutions are unbounded. That is to say that $\lim _{n \rightarrow \infty} F^{n}\left(x_{0}, y_{0}\right)=(K, \infty)$ and Theorem 3.1 fails since we have unbounded solutions.

Example 4.4 (Triangular Leslie-Gower Competition Model). Let us consider the triangular map $F: \mathbb{R}_{+}^{2} \rightarrow \mathbb{R}_{+}^{2}$ given by

$$
F(x, y)=\left(\frac{\mu K x}{K+(\mu-1) x}, \frac{\alpha L y}{L+(\alpha-1) y+\beta x}\right),
$$

where $\mu, \alpha>1, K, L>0$. This modified Leslie-Gower Competition Model has the following fixed points: $(0,0),(K, 0)$, $(0, L)$ and

$$
\left(x^{*}, y^{*}\right)=\left(K, \frac{(\alpha-1) L-\beta K}{(\alpha-1)}\right) .
$$

In order to have $\left(x^{*}, y^{*}\right)$ belonging in the first quadrant, let us assume that $(\alpha-1) L>\beta K$. We can also see that the map $F$ has a periodic orbit of prime period 2 whenever

$$
\left\{\begin{array}{l}
\frac{K \mu^{2} x}{K+\left(\mu^{2}-1\right) x}=x \\
\frac{\alpha^{2} L^{2} y}{(L+\beta x+(\alpha-1) y)\left(\frac{\beta K \mu x}{K+(\mu-1) x}+\frac{(\alpha-1) \alpha L y}{L+\beta x+(\alpha-1) y}+L\right)}=y .
\end{array}\right.
$$

From the first equation in the systems we get $x=0$ or $x=K$. If $x=0$, the solutions of the second equation are $y=0$ or $y=L$, i.e, we obtain the fixed points $(0,0)$ and $(0, L)$. If $x=K$, we obtain the solutions $y=0$ or $y=\frac{(\alpha-1) L-\beta K}{\alpha-1}$, that is, the fixed points $(K, 0)$ and $\left(x^{*}, y^{*}\right)$. Hence, the map $F$ has no periodic points of prime period two.

Notice that the orbits of $F$ are bounded since $\frac{\mu K x}{K+(\mu-1) x}<\frac{\mu K x}{(\mu-1) x}=\frac{\mu K}{\mu-1}$ and $\frac{\alpha L y}{L+(\alpha-1) y+\beta x}<\frac{\alpha L y}{(\alpha-1) y}=\frac{\alpha L}{\alpha-1}$.

Now, the Jacobian matrix of $F$ is given by

$$
D F(x, y)=J(x, y)=\left(\begin{array}{cc}
\frac{K^{2} \mu}{(K+x(\mu-1))^{2}} & 0 \\
-\frac{L y \alpha \beta}{(L+y(\alpha-1)+x \beta)^{2}} & \frac{L \alpha(L+x \beta)}{(L+y(\alpha-1)+x \beta)^{2}}
\end{array}\right) .
$$

Hence,

$$
J(0,0)=\left(\begin{array}{cc}
\mu & 0 \\
0 & \alpha
\end{array}\right), \quad J(K, 0)=\left(\begin{array}{cc}
\frac{1}{\mu} & 0 \\
0 & \frac{L \alpha}{L+K \beta}
\end{array}\right), \quad J(0, L)=\left(\begin{array}{cc}
\mu & 0 \\
-\frac{\beta}{\alpha} & \frac{1}{\alpha}
\end{array}\right),
$$


and

$$
J\left(x^{*}, y^{*}\right)=\left(\begin{array}{cc}
\frac{1}{\mu} & 0 \\
\frac{\beta(-\alpha L+L+K \beta)}{L(\alpha-1) \alpha} & \frac{L+K \beta}{L \alpha}
\end{array}\right) .
$$

A straightforward computation shows that the spectrum of $J(0,0), J(K, 0), J(0, L)$ and $J\left(x^{*}, y^{*}\right)$ all lie inside the unit disk. Consequently, the hypotheses of Theorem 3.1 are satisfied and every orbit converges to a fixed point.

Moreover, since $\left(x^{*}, y^{*}\right)=\left(K, \frac{(\alpha-1) L-\beta K}{\alpha-1}\right)$ is the unique fixed point in the interior of the first quadrant, it follows by Corollary 3.2 that $\left(x^{*}, y^{*}\right)$ is globally asymptotically stable fixed point with respect to the interior of the first quadrant.

Remark 4.5. It should be noted that $[8,9]$ investigated the global dynamics of Leslie-Gower model including the nonhyperbolic case.

Example 4.6 (Triangular Logistic-Type Map). Consider the triangular logistic-type map defined on $[0,1]^{N}$ given by

$$
F\left(x_{1}, x_{2}, \ldots, x_{N}\right)=\left(f_{1}\left(x_{1}\right), f_{2}\left(x_{1}, x_{2}\right), \ldots, f_{N}\left(x_{1}, x_{2}, \ldots, x_{N}\right)\right),
$$

where

$$
\begin{aligned}
& f_{1}\left(x_{1}\right)=\mu_{1} x_{1}\left(1-x_{1}\right), \\
& f_{2}\left(x_{1}, x_{2}\right)=\mu_{2} x_{2}\left(1-x_{2}\right) x_{1}, \\
& f_{3}\left(x_{1}, x_{2}, x_{3}\right)=\mu_{3} x_{3}\left(1-x_{3}\right) x_{1} x_{2},
\end{aligned}
$$

$$
f_{N}\left(x_{1}, x_{2}, \ldots, x_{N}\right)=\mu_{N} x_{N}\left(1-x_{N}\right) \prod_{i=1}^{N-1} x_{i},
$$

where $\mu_{i}>0$, for all $i \in\{1,2, \ldots, N\}$. The map $F$ has the following $N+1$ fixed points

$$
\begin{aligned}
& E_{0}=(0,0, \ldots, 0), \quad E_{1}=\left(e_{1}^{*}, 0, \ldots, 0\right), \quad E_{2}=\left(e_{1}^{*}, e_{2}^{*}, 0, \ldots, 0\right), \ldots \\
& E_{N-1}=\left(e_{1}^{*}, e_{2}^{*}, \ldots, e_{N-1}^{*}, 0\right), \quad E_{N}=\left(e_{1}^{*}, e_{2}^{*}, \ldots, e_{N-1}^{*}, e_{N}^{*}\right),
\end{aligned}
$$

where

$$
e_{1}^{*}=\frac{\mu_{1}-1}{\mu_{1}}, \quad e_{2}^{*}=\frac{\sum_{j=1}^{2}\left(\prod_{i=1, i \neq j}^{2} \mu_{i}\right)-\prod_{i=1}^{2} \mu_{i}}{\mu_{2}\left(1-\mu_{1}\right)}
$$

and

$$
e_{k}^{*}=\frac{\sum_{j=1}^{k}\left(\prod_{i=1, i \neq j}^{k} \mu_{i}\right)-\prod_{i=1}^{k} \mu_{i}}{\left(\sum_{j=1}^{k-1}\left(\prod_{i=1, i \neq j}^{k-1} \mu_{i}\right)-\prod_{i=1}^{k-1} \mu_{i}\right) \mu_{k}}, \quad k=3,4, \ldots, N .
$$

The spectrum of the Jacobian matrix of $F$ evaluated at the fixed points are

$$
\begin{aligned}
& \sigma_{E_{0}}=\left\{\mu_{1}, 0, \ldots, 0\right\} \\
& \sigma_{E_{1}}=\left\{2-\mu_{1},-e_{1}^{*} \mu_{2}, 0, \ldots, 0\right\} \\
& \sigma_{E_{2}}=\left\{2-\mu_{1}, 2-e_{1}^{*} \mu_{2},-e_{1}^{*} e_{2}^{*} \mu_{3}, 0, \ldots, 0\right\} \\
& \vdots \\
& \sigma_{E_{N-1}}=\left\{2-\mu_{1}, 2-e_{1}^{*} \mu_{2}, 2-e_{1}^{*} e_{2}^{*} \mu_{3}, \ldots, 2-\left(\prod_{i=1}^{N-2} e_{i}^{*}\right) \mu_{N-1},-\left(\prod_{i=1}^{N-1} e_{i}^{*}\right) \mu_{N}\right\} \\
& \sigma_{E_{N}}=\left\{2-\mu_{1}, 2-e_{1}^{*} \mu_{2}, 2-e_{1}^{*} e_{2}^{*} \mu_{3}, \ldots, 2-\left(\prod_{i=1}^{N-2} e_{i}^{*}\right) \mu_{N-1}, 2-\left(\prod_{i=1}^{N-1} e_{i}^{*}\right) \mu_{N}\right\} .
\end{aligned}
$$

By considering parameters values as shown in Table 1, we know that each fixed point $E_{i}$ is locally asymptotically stable. Let us now look how Theorem 3.1 establishes the global dynamics of the fixed points $E_{i}, i=0,1, \ldots, N$. Indeed, in order to apply Theorem 3.1, it suffices to show that there are no periodic points. 


\section{Table 1}

Regions, in the parameter space, of local stability of the fixed points $E_{i}$,

$i=0,1, \ldots, N$ of the triangular logistic map.

\begin{tabular}{ll}
\hline Fixed point & Region of local stability \\
\hline$E_{0}$ & $\mu_{1} \leq 1$ \\
$E_{1}$ & $1<\mu_{1} \leq 3, e_{1}^{*} \mu_{2} \leq 1$ \\
$E_{2}$ & $1<\mu_{1} \leq 3,1<e_{1}^{*} \mu_{2} \leq 3, e_{1}^{*} e_{2}^{*} \mu_{3} \leq 1$ \\
$\vdots$ & $\vdots$ \\
$E_{N-1}$ & $\left\{\begin{array}{l}1<\mu_{1} \leq 3 \\
1<\left(\prod_{i=1}^{k-1} e_{i}^{*}\right) \mu_{k} \leq 3, \text { for all } k \in\{2,3, \ldots, N-1\} \\
\left(\prod_{i=1}^{N-1} e_{i}^{*}\right) \mu_{N} \leq 1\end{array}\right.$ \\
$E_{N}$ & $\left\{\begin{array}{l}1<\mu_{1} \leq 3 \\
1<\left(\prod_{i=1}^{k-1} e_{i}^{*}\right) \mu_{k} \leq 3, \text { for all } k \in\{2,3, \ldots, N\}\end{array}\right.$ \\
\hline
\end{tabular}

The map $F$ has a 2-periodic orbit when

$$
\left\{\begin{array}{l}
f_{1}\left(f_{1}\left(x_{1}\right)\right)=x_{1} \\
f_{2}\left(f_{1}\left(x_{1}\right), f_{2}\left(x_{1}, x_{2}\right)\right)=x_{2} \\
f_{3}\left(f_{1}\left(x_{1}\right), f_{2}\left(x_{1}, x_{2}\right), f_{3}\left(x_{1}, x_{2}, x_{3}\right)\right)=x_{3} \\
\vdots \\
f_{N}\left(f_{1}\left(x_{1}\right), \ldots, f_{N}\left(x_{1}, \ldots, x_{N}\right)\right)=x_{N} .
\end{array}\right.
$$

The solutions of the first equation are $x_{1}^{1}=0, x_{1}^{2}=e_{1}^{*}$ and

$$
x_{1}^{3,4}=\frac{1+\mu_{1} \pm \sqrt{\left(\mu_{1}-3\right)\left(\mu_{1}+1\right)}}{2 \mu_{1}} .
$$

Now, if $\mu_{1}<3$, then there are no real solutions to (7). When $\mu_{1}=3$ the solutions to (7) are $x_{1}^{3}=x_{1}^{4}=\frac{2}{3}=e_{1}^{*}$. Hence, when $\mu_{1} \leq 3, f_{1}$ has only fixed points 0 and $e_{1}^{*}$.

Now, suppose that $1<\mu_{1} \leq 3$ and $x_{1}=e_{1}^{*}$. Then the second equation in (6) is equivalent to

$$
\left(\mu_{2} e_{1}^{*}\right)^{2} x_{2}\left(1-x_{2}\right)\left(1-\mu_{2} e_{1}^{*} x_{2}\left(1-x_{2}\right)\right)=x_{2} .
$$

The solutions of this equation are $x_{2}^{1}=0, x_{2}^{2}=e_{2}^{*}$ and

$$
x_{2}^{3,4}=\frac{1+\mu_{2} e_{1}^{*} \pm \sqrt{\left(\mu_{2} e_{1}^{*}-3\right)\left(\mu_{2} e_{1}^{*}+1\right)}}{2 \mu_{2} e_{1}^{*}} .
$$

Again, if $\mu_{2} e_{1}^{*}<3$, then there are no real solutions to (8). When $\mu_{2} e_{1}^{*}=3$ the solutions of (8) are $x_{2}^{3}=x_{2}^{4}=\frac{2}{3}=e_{2}^{*}$. Consequently, the map $f_{2}$ has no periodic points, only fixed points.

Now, suppose that $1<\mu_{1} e_{1}^{*} \leq 3$ and $x_{2}=e_{2}^{*}$. Then the third equation in (6) simplifies to

$$
\left(\mu_{3} e_{1}^{*} e_{2}^{*}\right)^{2} x_{3}\left(1-x_{3}\right)\left(1-\mu_{2} e_{1}^{*} e_{2}^{*} x_{3}\left(1-x_{3}\right)\right)=x_{3},
$$

where the solutions are $x_{3}^{1}=0, x_{3}^{2}=e_{3}^{*}$ and

$$
x_{3}^{3,4}=\frac{1+\mu_{2} e_{1}^{*} e_{2}^{*} \pm \sqrt{\left(\mu_{2} e_{1}^{*} e_{2}^{*}-3\right)\left(\mu_{2} e_{1}^{*} e_{2}^{*}+1\right)}}{2 \mu_{2} e_{1}^{*} e_{2}^{*}} .
$$

Using the same argument, if $\mu_{2} e_{1}^{*} e_{2}^{*}<3$, then there are no real solutions to (9). Hence, $x_{3}$ in (9) are impossible and will not be in account. When $\mu_{2} e_{1}^{*} e_{2}^{*}=3$, the solutions of (9) are $x_{3}^{3}=x_{3}^{4}=\frac{2}{3}=e_{3}^{*}$. This implies that the map $f_{3}$ has no periodic points, only fixed points.

Repeating this process one may conclude that the map $F$ has no periodic points of minimal period 2 if $\mu_{1} \leq 3$ and $\left(\prod_{i=1}^{k-1} e_{i}^{*}\right) \mu_{k} \leq 3$, for all $k \in\{2,3, \ldots, N\}$.

Moreover, if we assume that

$$
\left\{\begin{array}{l}
1<\mu_{1}<3 \\
1<\left(\prod_{i=1}^{k-1} e_{i}^{*}\right) \mu_{k}<3, \quad \text { for all } k \in\{2,3, \ldots, N\},
\end{array}\right.
$$


then all fixed points $E_{k}$ are hyperbolic and the hypotheses of Theorem 3.1 are verified. Hence, we conclude that every orbit converges to a fixed point.

Finally, by Corollary 3.2 the fixed point $E_{N}$ is globally asymptotically stable with respect to the interior of [0,1] $]^{N}$ since it is the unique fixed point in the interior of $[0,1]^{N}$.

Remark 4.7. It is worthwhile to observe that most of the maps we have considered are not monotone in the sense of Smith [6]. In fact, with the exception of Example 4.4, the maps in the above examples are not monotone.

\section{Acknowledgment}

We would like to thank Michal Misiurewicz for our useful discussions and for providing interesting examples that improved the presentation of this paper.

\section{References}

[1] J.P. LaSalle, The Stability of Dynamical Systems, SIAM, Philadelphia, 1976.

[2] Marc Chamberland, Dynamics of maps with nilpotent Jacobians, J. Difference Equ. Appl. 12 (1) (2006) 49-56.

[3] Mario Martelli, Global stability of stationary states of discrete dynamical systems, Ann. Sci. Math. Québec 22 (2) (1998) 201-212. Dedicated to the memory of Gilles Fournier (Sherbrooke, PQ, 1997).

[4] E.C. Balreira, S. Elaydi, R. Luís, Local stability implies global stability for the planar Ricker competition model, Discrete Contin. Dyn. Syst. 19 (2) (2014) 323-351.

[5] S. Elaydi, R. Sacker, Basin of attraction of periodic orbits of maps on the real line, J. Difference Equ. Appl. 10 (10) (2004) $881-888$.

[6] H.L. Smith, Planar competitive and cooperative difference equations, J. Difference Equ. Appl. 3 (5-6) (1998) 335-357.

[7] M. Kulenović, O. Merino, Competitive-exclusion versus competitive-coexistence for systems in the plane, Discrete Contin. Dyn. Syst. Ser. B 6 (2006) 1141-1156.

[8] M. Kulenović, O. Merino, Global bifurcations for competitive systems in the plane, Discrete Contin. Dyn. Syst. Ser. B 12 (2009) 133-149.

[9] M. Kulenović, O. Merino, Invariant manifolds for competitive discrete systems in the plane, Int. J. Bifurcation Chaos 20 (2010) $2471-2486$.

[10] A. Amleh, E. Camouzis, G. Ladas, On the dynamics of a rational difference equation, Part I, Int. J. Difference Equ. 3 (2008) 1-35.

[11] A.N. Sharkovsky, Coexistence of cycles of a continuous map of the line into itself, Int. J. Bifurcation Chaos 05 (05) (1995) 1263-1273.

[12] P. Kloeden, On Sharkovsky's cycle coexistence ordering, Bull. Aust. Math. Soc. 20 (1979) 171-177.

[13] M. Guzowska, R. Luís, S. Elaydi, Bifurcation and invariant manifolds of the logistic competition model,J. Difference Equ. Appl. 17 (12)(2011) 1851-1872.

[14] S. Elaydi, Discrete Chaos: With Applications in Science and Engineering, second ed., Chapman and Hall/CRC, Boca Raton, 2008.

[15] Clark Robinson, Dynamical Systems: Stability, Symbolic Dynamics, and Chaos, second ed., CRC Press, Inc., Boca Raton, 1999.

[16] P. Brunovský, P. Poláčik, On the structure of $\omega$-limit sets of maps, Z. Angew. Math. Phys. 48 (1997) $976-986$.

[17] Bernd Aulbach, Iterates approaching hyperbolic manifolds of fixed points, J. Reine Angew. Math. 350 (1984) 68-86.

[18] S. Elaydi, An Introduction to Difference Equations, third ed., Springer, New York, 2005.

[19] R. Luís, S. Elaydi, H. Oliveira, Stability of a Ricker-type competition model and the competitive exclusion principle, J. Biol. Dyn. 5 (6) (2011) 636-660. 